\title{
ATYPICAL IMAGING CHARACTERISTIC OF OLIGODENDROGLIOMA
}

${ }^{1}$ Baduku TS and ${ }^{2}$ Shokunbi T

${ }^{1}$ Department of Radiology Kaduna State University, Kaduna ${ }^{2}$ Department of Surgery, University of Ibadan, Ibadan

\begin{abstract}
Background: There has been the dearth of doctors choosing the postgraduate specialties that were outside the core clinical disciplines. This would no longer impact negatively in the distribution of Doctors in the less sought out specialties in terms of service delivery and medical education.
\end{abstract}

Objectives: The present study was done to determine the career preferences of final year medical students specifically as regards to their choice of chemical pathology and factors influencing such choice.

Methods: The study was a Descriptive cross sectional study. A questionnaire was self administered by the authors on all the final year medical students of Usmanu Danfodio University, Sokoto. Data was analyzed with SPSS version 20.0

Results: Out of the 60 respondents only 3(5\%) had interest in pursuing chemical pathology as a postgraduate medical career. The medical students with ages ranging from 20 to 24 years were more likely to choose chemical pathology as a career using the multivariate model $(\mathrm{OR}=25.692,95 \% \mathrm{CI}=7.125-100.577)$.

Correspondence: Dr Tokan Silas Baduku Department of Radiology Kaduna State University, Kaduna Email : tokanbaduku@yahoo.com Tel: +2348023612828
Conclusion: It is paramount on all stakeholders of medicine, to improve the profile and change the misconceptions about Chemical Pathology. This would help to improve the practice of medicine in general.

Key words: Career choice, Usmanu Danfodiyo University, Sokoto, Reasons for specialization

\section{INTRODUCTION}

$\mathrm{B}$ rain tumours are either primary or metastases from distant sites. Of the primary brain tumours, the commonest arise from the glial cells that make and maintain the central nervous system. ${ }^{1}$ These tumour cells, commonly called gliomas, are malignant and account for $30-40 \%$ of all primary intracranial tumours. ${ }^{2}$ Of all the gliomas, oligodendrogliomas are the least invasive. ${ }^{3}$ They are clinically slow growing and may run a course of 5 to 7 years. ${ }^{4}$

Presented below is a case of a teenage girl who's presenting features coincided with a road traffic accident, there by swaying the neurosurgeons into making an initial diagnosis of intracranial head injury.

\section{CASE REPORT}

A.A is a 15-year old girl who presented to our hospital with history of gradual loss of vision for six months, difficulty with speech, weakness of the left upper and lower limbs, swollen vertex of the head, and irrational behavior of two weeks duration following road traffic accident in which she fell off a moving motor circle. She was said to have landed with the head and sustained bruises but there was no history of seizure disorders or loss of consciousness. She was treated as an outpatient in a private hospital but the symptoms persisted. Examination revealed the main findings in the central nervous system. There was a mildly tender soft tissue swelling over the vertex, measuring $3 \mathrm{~cm} \times 5 \mathrm{~cm}$. The muscle power in both left upper and lower limbs was grade 3 while the contra lateral side was normal. There was increased muscle tone of the left upper and lower limbs. Her speech was also slurred.

Skull radiographs showed an amorphous dense opacity in the right fronto-parietal region. There were thumb printing impressions on the calvarium with sutural diastasis of the anterior 
fontanelle and erosion of the posterior clinoid processes. A diagnosis of intracranial space occupying lesion with associated raised intracranial pressure was made. Computed tomography of the brain showed well marginated calcific mass measuring $5.3 \mathrm{~cm}$ and $7.0 \mathrm{~cm}$ in size in the right fronto-temporo-parietal region (Fig. 1). There were surrounding areas of hypodensity, in keeping with cerebral oedema. There was also thinning, with irregularity of the parietal bone overlying the area of mass lesion. There was mass effect on the opposite side. Based on these radiological findings, oligodendroglioma was suspected. However, the differential diagnoses included meningioma, calcified dermoid, craniopharyngioma and calcified tuberculoma.

The patient had craniectomy done with extensive excision of the tumour and the histology showed an anaplastic oligodendroglioma of the right frontal lobe. The patient was later placed on steroids and had radiotherapy. Post-operative scan showed a small calcified residual tumour. The patient was discharged home to come back for re-excision of the residual tumour but was lost to follow-up.

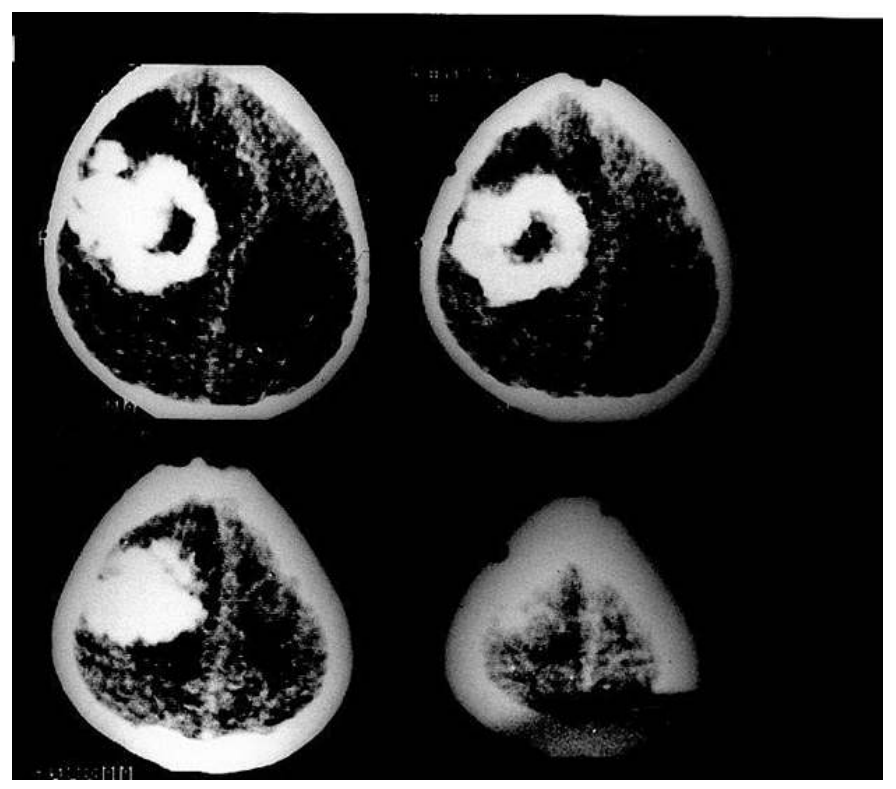

Fig.1.Bone window CT scan showing ring-like highly calcified mass in the right tempero-parietal area with mass effect to the contralateral side.

\section{DISCUSSION}

Oligodendrogliomas are primary intracranial tumours which are relatively rare, accounting for 2 to $5 \%$ of all neoplasms, and 5 to $10 \%$ of intracranial gliomas. ${ }^{3}$ Oligodendeogliomas are tumours of adults, occurring mainly in middle age group of $4-5^{\text {th }}$ decades of life, with no sex predilection. ${ }^{5}$ The ratio of adults to children with oligodendroglioma is $8: 1$ with a peak incidence of $35-45$ years. ${ }^{5}$ They are also rare in teenagers, though a study by Dickson et $a l^{7}$ showed a reduced incidence of gliomas from $1.4 \%$ to $0.8 \%$. This patient was only a 15 year old female when she presented to our medical facility.

Oligodendrogliomas are primarily supratentorial tumours, with $85 \%$ occurring in this area. ${ }^{8}$ They have a predilection for the cerebral hemispheres, with most common location in the frontal lobes. ${ }^{9}$ Although they can be found anywhere, the posterior fossa is an uncommon site. ${ }^{1}$ Cortical involvement is very uncommon while a purely intraventricular tumour is also rare. ${ }^{10}$

World Health Organization classified oligodendrogliomas into 3 types, namely the 'pure' type, the 'mixed' type, and the 'anaplastic' type. ${ }^{4}$ Although oligodendrogliomas are slow growing tumouts, a minority are anaplastic. ${ }^{5}$ This anapla tic type, which this patient has is occasionally encountered and may undergo malignant transformation to a glioblastoma multiforme. ${ }^{3}$ Whatever the histological type, oligod nndrogliomas generally calcify. Of the 6-10 fo radiographically visible calcififations seen in intracranial tumours, oligodфndrogliomas constitute a significant percentage. ${ }^{1}$ Calcification occurs in about $72 \%$ f cases of oligodendrogliomas, ${ }^{11}$ althou $\$$ cases that are entirely free from calcifiqation have been reported. ${ }^{12}$

Computed tomography will normally show nodular of clumped calcifications in about 70$90 \%$ of fases but the calcification may be peripheral. ${ }^{4}$ From time to time, 
oligodendrogliomas may infiltrate the meninges and finally cause some slight destruction of the overlying bone, causing scalloping of the inner table. ${ }^{3}$ This patient showed clumped calcification with erosion of both inner and outer tables of the cranial vault. About two-thirds of oligodendrogliomas show mild to moderate enhancement following contrast administration. ${ }^{11}$

Computed tomography is very valuable in the diagnosis of calcification in intracranial tumours. This is because it has a higher sensitivity compared to plain skull radiographs alone. However, it is not specific in the diagnosis of oligodendrogliomas. Other intracranial tumours that frequently give roentgenologically visible calcification simulating oligodendrogliomas are astrocytomas, meningiomas, calcified dermoids, teratomas, pituitary adenomas, craniopharyngiomas and calcified tuberculomas. Generally, oligodendromas show higher levels of calcifications, with surrounding oedema. ${ }^{4}$ In this case, histology gave the definitive diagnosis. Again computed tomography is essential in the follow up of patients, whether post-operative, postradiotherapy or during chemotherapy, since it shows tumour regression or any other complication. In this patient, the post-operative brain scan showed residual tumour which was to be excised at a later date if the patient had not defaulted.

\section{CONCLUSION}

The case of anaplastic oligodendroglioma in a 15 year-old girl, who presented to our hospital following road traffic accident, is hereby reported. The diagnosis was suggested by computed tomographic scan and confirmed by histology.

\section{REFERENCES}

1. Ricci PE, Dungan,DH. Imaging of low- and intermediate-grade gliomas.Semin. Radiat. Oncol.2001; 11(2): 103-112.

2. Runge VM, Clinical Magnetic Resonance Imaging 2001. JB Saunders (W.B) Co. Ltd. Pp: 124-126.
3. Osborn AG, Osborn's Brain: Imaging, Pathology and Anatomy. Lippincot, Williams and Wilkins. Diagnostic Neuroradiology. 2012; 402-410

4. Louis DN, Ohgaki H, Wieslter OD, Cavenee WK, Peter CB, Jouvet A, Scheithauer BW, Kleihues P. The 2007 classification of tumours of the CNS. Acta Neuropathol. 2007; 114:97-107

5. Malene SN, Helle CC. Incidence of and survival from oligodendroglioma in Denmark. Neuro. Oncol. 11 (3):311-317

6. Alston RD, Geraci M, Eden TOB, MoranA, Rowan S, Birch JM. Changes in Cancer incidence in teenagers and young adults (Ages 13-24 years) in England: 19792003. Cancer 2008; 113 (10) : 2807-2815.

7. Dickson PV, Davidoff AM. Malignant neoplasms of the head and neck. Semin. Pediatr. Surg. 2006; 15: 92-98

8. Shokry L, Hariri OR, Miulli DE, Amin J, Minasian T, Gupta RK, Sidiggi J. Supratentorial primitive neuroectodermal tumour in an adult: a case report and review of the literature. Journal of Medical case Reports. 2012; 6:361-365.

9. Porjazova E, Spasov SS, Stefanova P, P o r j a z o a R. M a lig n a t Oligodendroglioma in a child: A case report. Afri. J. of Ped. Surgery 2005; 2(1); 32-34.

10. Igun GO. Diagnosis and management of brain tumours at Jos University Teaching Hospital, Nigeria. East African Medical Journal. 2001; 78(3): 148-151.

11. Tamura M, Zama A, Kurihara H, Kano T, Imai H, Ishiuchi, IwaiT, NaitoI. Clinicohistological study of oligodendrogliomas and oligoastrocytoma. Brain Tumor Pathol. 1997; 14:35-39.

12. ScheitheuerBW. Brain Pathol. Intl. Society of Neuropath. 2009; 19 (4): 551-564. 